\title{
Magnetic Heating of Nanoparticles Applied in the Synthesis of a Magnetically Recyclable Hydrogenation Nanocatalyst
}

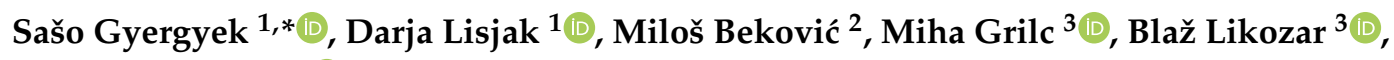 \\ Marijan Nečemer ${ }^{4}\left(\mathbb{D}\right.$ and Darko Makovec ${ }^{1}$ \\ 1 Department for Materials Synthesis, Jožef Stefan Institute, Jamova 60, 1000 Ljubljana, Slovenia; \\ darja.lisjak@ijs.si (D.L.); darko.makovec@ijs.si (D.M.) \\ 2 Institute of Electrical Power Engineering, Faculty of Electrical Engineering and Computer Science, \\ University of Maribor, Koroška 46, 2000 Maribor, Slovenia; milos.bekovic@um.si \\ 3 Department of Catalysis and Chemical Engineering, National Institute of Chemistry, Hajdrihova 19, \\ 1000 Ljubljana, Slovenia; miha.grilc@ki.si (M.G.); blaz.likozar@ki.si (B.L.) \\ 4 Department for Low and Medium Energy Physics, Jožef Stefan Institute, Jamova 60, 1000 Ljubljana, Slovenia; \\ marijan.necemer@ijs.si \\ * Correspondence: saso.gyergyek@ijs.si
}

Received: 29 April 2020; Accepted: 3 June 2020; Published: 10 June 2020

\begin{abstract}
Utilization of magnetic nanoparticle-mediated conversion of electromagnetic energy into heat is gaining attention in catalysis as a source of heat needed for a substrate's chemical reaction (electrification of chemical conversions). We demonstrate that rapid and selective heating of magnetic nanoparticles opens a way to the rapid synthesis of a nanocatalyst. Magnetic heating caused rapid reduction of $\mathrm{Ru}^{3+}$ cations in the vicinity of the support material and enabled preparation of a Ru nanoparticle-bearing nanocatalyst. Comparative synthesis conducted under conventional heating revealed significantly faster $\mathrm{Ru}^{3+}$ reduction under magnetic heating. The faster kinetic was ascribed to the higher surface temperature of the support material caused by rapid magnetic heating. The nanocatalyst was rigorously tested in the hydrotreatment of furfural. The activity, selectivity and stability for furfural hydrogenation to furfuryl alcohol, a valuable biobased monomer, remained high even after four magnetic recycles.
\end{abstract}

Keywords: catalyst; ruthenium; biomass; induction heating; magnetic separation; magnetic heating

\section{Introduction}

Renewable electricity will play an important role in the near future decarburization of human society. Due to variable availability, new strategies for its use must be developed in addition to storage in batteries. The direct conversion of surplus renewable electricity into chemicals such as fuels, monomers, solvents etc., a concept that the German Chemical Engineering Association named power-to-chemicals (P2C), is becoming an important additional strategy [1]. The current focus is on electrocatalytic processes [1]; however, it makes sense to develop other electricity-based technologies. Magnetic nanoparticles (MNPs) heat rapidly and extensively under the influence of an alternating magnetic field, a phenomenon extensively studied for cancer treatment [2,3]. The application of this principle to drive chemical reactions has only recently been demonstrated [4-10]. Kirschning et al. showed for the first time that magnetic nanoparticles in the Alternating Current (AC) field of low-to-medium frequency can heat sufficiently to drive technologically important chemical reactions in continuous operation and significantly improve yields compared to conventional heating [4]. Chaudret et al. developed complex MNP-bearing catalytic Ru nanoparticles with superior magnetic properties, 
resulting in an exceptionally high specific absorption rate (SAR), the figure-of-merit indicating the amount of heat the material generates in the AC field of the specified frequency and amplitude $[7,8]$. Complex nano-objects have been used to demonstrate magnetically induced batch $\mathrm{CO}$ and continuous $\mathrm{CO}_{2}$ hydrogenation $[7,8]$. It has been pointed out recently that the ability to use an AC field to deliver heat where needed is an enabling technology suited to push many catalytic reactions beyond the reactor heat transfer limits, to the limits of process kinetics [9]. Overall, the claimed advantages of the technology are manifold: more favorable energy balance, process intensification, reactor setup simplification, reduced safety issues, minor operational costs and increased process productivity [9]. In addition to novel applications in catalysis, magnetic induction heating has been proven in the synthesis of metal-organic framework MNP composites (MOF-MNPs) [11] and in its application in magnetic induction swing adsorption (MISA) [12]. Magnetically induced selective heating of MNPs dispersed in the MOF precursor solution has shown an advantage in the increased kinetics of formation of MOF over conventional heating [11]. Furthermore, the yield and size of composite MOFs can be controlled by reaction time, MNP concentration and strength of magnetic field [11]. Supported precious metal nanoparticles, such as ruthenium $(\mathrm{Ru})$ nanoparticles, are often used in the catalytic conversion of biomass into value-added chemicals [13]. Carbon as a support material for catalysts has long been relevant due to its commercial availability, thermochemical stability, low density and simple preparation from various natural sources, such as biomass, sugars and fatty acids [14,15]. Catalytic nanoparticles are typically deposited on a support material using methods that fall broadly into two categories: impregnation of support [16] and precipitation in suspension of the support material $[16,17]$. The methods of the first category are based on adsorption of metal cations on the surface of the support from the solution, followed by drying and reduction, or on adsorption of previously synthesized metal nanoparticles. Methods from the second category are based on the reduction of the metal precursor in the suspension of the support, causing the precipitation of metal nanoparticles and their deposition on the support's surface. Recently, our group has developed a method for preparation of magnetically separable Ru-based nanocatalysts that shows remarkable activity for the hydrogenation/hydrodeoxygenation of the lignin monomer model compound eugenol $[17,18]$. The method is based on solvothermal reduction of $\mathrm{Ru}(\mathrm{acac})_{3}$ in the suspension of the magnetic carbon support by the environmentally benign solvent isopropanol.

Here, we applied for the first time the induction heating of magnetic nanoparticles incorporated within the graphitic material (support) to demonstrate the synthesis of a nanocatalyst. Specifically, induction heating of the magnetic nanoparticles caused the surface temperature of the support to rise rapidly, while the bulk of the liquid remained at a much lower temperature. A high enough temperature was achieved to cause a rapid reduction of $\mathrm{Ru}^{3+}$ and an exclusive deposition of $\mathrm{Ru}$ nanoparticles and atomic clusters on the surface of the support. The nanocatalyst showed a $100 \%$ conversion of furfural with $99 \%$ selectivity for valuable biobased monomer furfuryl alcohol, even after being recycled four times.

\section{Materials and Methods}

Iron (III) sulphate hydrate $\left(\mathrm{Fe}_{2}\left(\mathrm{SO}_{4}\right)_{3} x \mathrm{H}_{2} \mathrm{O}\right.$, (Sigma-Aldrich, St. Louis, $\mathrm{MO}$, USA), iron (II) sulphate hydrate $\left(\mathrm{FeSO}_{4} \cdot 7 \mathrm{H}_{2} \mathrm{O} 92 \%\right.$, Alfa-Aesar, Haverhill, $\left.\mathrm{MA}, \mathrm{USA}\right)$, ammonium hydroxide $\left(\mathrm{NH}_{4} \mathrm{OH} 25 \%\right.$ solution, J.T. Baker, Radnor Township, PA, USA), citric acid monohydrate (ACS 99-102\%, Alfa-Aesar), acetone (reagent grade, Carlo-Erba, Barcelona, Spain), glucose (d-glucose 99\%, Alfa-Aesar), ruthenium (III) 2,4-pentadionate $\left(\mathrm{Ru}\left(\mathrm{C}_{5} \mathrm{H}_{7} \mathrm{O}_{2}\right)_{3} 97 \%\right.$, Sigma-Aldrich), 2-propanol (ACS reagent grade, VWR, Radnor, PA, USA), Ru/C (5 wt.\% Ru, Sigma Aldrich) and furfural (99\%, Sigma-Aldrich) were used as received.

The magnetic nanoparticle-containing support (MN-C) was prepared by modification of our previously reported route (see Supplementary Materials Section S1 for details) [17]. In short, the precursor particles were prepared by hydrothermal treatment of glucose dissolved in the aqueous suspension of citric-acid-coated magnetic iron oxide nanoparticles [17]. The precursor particles 
had a diameter of approximately 100-300 $\mathrm{nm}$ and contained homogeneously dispersed iron oxide nanoparticles ( $10 \mathrm{~nm}$ in diameter) in the carbonaceous matrix [17]. The MN-C was prepared by annealing the precursor for $6 \mathrm{~h}$ at $600{ }^{\circ} \mathrm{C}$ in an Ar atmosphere. Magnetically mediated synthesis of the catalyst AC-Ru was conducted in a round bottom glass pressure vessel (see Supplementary Materials Section S1 for details, Table S1). The MN-C and $3 \mathrm{~mL}$ of $0.01 \mathrm{M} \mathrm{Ru}$ (III) 2,4-pentadionate were sealed, the vessel was placed in the centre of the inductor coil and the field of $\mu_{0} H=86 \mathrm{mT}$ was turned on. After $19 \mathrm{~min}$, the vessel was removed from the coil and in approximately $3 \mathrm{~min}$ cooled to room temperature when it was opened and the AC-Ru was separated using a handheld permanent magnet. The AC-Ru was washed 5 times with pure 2-propanol and dried in a vacuum at room temperature. The catalyst $\mathrm{CH}-\mathrm{Ru}$ was synthesized under identical conditions, but using an oil bath heated to $150{ }^{\circ} \mathrm{C}$ as heat source. The synthesis proceeded for $30 \mathrm{~min}$ (see Supplementary Materials Section S1 for details). The hydrogenation of furfural was conducted in a pressure vessel (see Supplementary Materials Section $\mathrm{S} 1$ for details). Reaction conditions: $48 \mathrm{mg}$ of catalyst, $0.1 \mathrm{~g}$ of furfural, $0.8 \mathrm{~g}$ of 2-propanol, $\mathrm{H}_{2}$ pressure $10 \mathrm{bar}, 90^{\circ} \mathrm{C}$ for $3 \mathrm{~h}$. The AC-Ru catalyst was recycled 4 times (5 hydrogenations in total using the same catalyst) and the $\mathrm{CH}-\mathrm{Ru} 1$ time (2 hydrogenations in total using the same catalyst).

Scanning electron microscope (SEM) images of the AC-Ru were obtained using a Jeol JSM-7600F (Jeol, Tokyo, Japan) microscope. The sample was deposited on a conducting graphite tape and imaged without any further preparation. The X-ray powder diffraction pattern (XRD) was collected using a Siemens D5005 diffractometer (Siemens, Munich, Germany) with a monochromator in the diffracted beam. Quantitative phase analyses based on a Rietveld refinement of the XRD pattern of the MN-C were

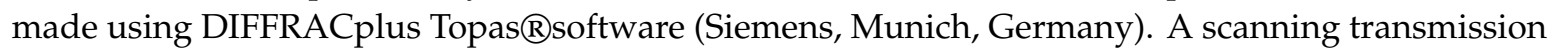
electron microscope (STEM) Cs-corrected Jeol ARM 200CF STEM (Jeol, Tokyo, Japan) operated at $80 \mathrm{kV}$ was used to observe the AC-Ru and CH-Ru catalysts, deposited on a copper-grid-supported lacy carbon foil. During the analysis, High Angle Annular Dark Field (HAADF) and Bright Field (BF) detectors were used simultaneously at 68-180 and 10-16 mrad collection semi-angles, respectively. The Raman spectrum of the MN-C was recorded with a NT-MDT model Integra Spectra for Materials Science equipped with a confocal microscope (20× magnification) at room temperature. A 633-nm laser diode was used for the excitation. The scattered light was detected using a cooled Charge Coupled Device (CCD) camera and a 600 grooves/mm grating, and 10 spectra were accumulated, each acquired for $60 \mathrm{~s}$ to increase the signal-to-noise ratio. The spectra were analyzed offline using OriginPro 2015 64-bit software (OriginLabs, Northampton, MA, USA). Raman spectra were fitted with 5 Lorentzian functions. Nitrogen adsorption/desorption isotherm was measured for the sample at liquid-nitrogen temperature using a Nova 2000e (Quantachrome, Boynton Beach, FL, USA) nitrogen-sorption analyzer. Prior to the measurement, the sample was degassed over night at $120^{\circ} \mathrm{C}$ in a vacuum. The surface area was calculated using the Brunauer-Emmett-Teller (BET) equation with the nitrogen-adsorption data in the $P / P_{0}$ range between 0.05 and 0.3 (7-point analysis). The room-temperature magnetization curve of the MN-C was measured with a vibrating-sample magnetometer (VSM) LakeShore 7307 VSM. Ru and Fe contents in MN-C, AC-Ru and $\mathrm{CH}-\mathrm{Ru}$ were non-destructively determined using an energy dispersive $\mathrm{X}$-ray fluorescence spectrometer (XRF) comprised of a Ge semiconductor detector (GLP-16195/10-P, ORTEC, Oak Ridge, TN, USA) with an energy resolution of $401 \mathrm{eV}$ at $60 \mathrm{keV}$, a spectroscopy amplifier (M2020, Canberra, Meriden, CT, USA), an Analogue Digital Converter (M8075, Canberra) and a Personal Computer-based Multi Chanel Analyzer (S-100, Canberra). For the excitation, the annular Am-241 radioactive source ( $25 \mathrm{mCi}$, Isotope Products Laboratories, Santa Clara, CA, USA) was used. Quantification was performed utilizing the in-house developed QAES (quantitative analysis of environmental samples) software [19]. For the analysis, sample powders were diluted with cellulose and pressed into a pellet of $24 \mathrm{~mm}$ in diameter. The specific absorption rate (SAR) of the MN-C was determined from the measurements of temperature increase in the AC field. An amount of $0.25 \mathrm{~mL}$ of the suspension of $5 \mathrm{wt} . \% \mathrm{MN}-\mathrm{C}$ in isopropanol was placed in a plastic round-bottom 2-mL Eppendorf tube. The tube that was isolated from the surroundings by a polystyrene chamber was fitted in the center of the coil (Figures S1 and S2). The temperature at the middle of the sample was continuously 
measured every second using a fiber optical probe. The sample was exposed to the AC field for a time varying between 15 and $20 \mathrm{~s}$. The heating curves were fitted with a linear function in the initial region where the increase in temperature was linear with time. SAR values were calculated using the expression:

$$
S A R=\frac{c_{p}}{w}\left(\frac{\mathrm{d} T}{\mathrm{~d} t}\right)_{i}
$$

where $c_{p}$ is the heat capacity of isopropanol $(2.68 \mathrm{~J} / \mathrm{gK}), w$ is the weight fraction of Fe in the MN-C in isopropanol and $(\mathrm{d} T / \mathrm{d} t)_{\mathrm{I}}$ is the slope of the linear fit in the initial region. The SAR can be interpreted as the amount of heat generated by the sample per gram of Fe incorporated within the MN-C was calculated, where the weight fraction of Fe in MN-C was determined by the XRF. Liquid products of the hydrogenations were analyzed by Gas Chromatography (GC-QMS) (Ultra 2010, Shimadzu, Kyoto, Japan) and ${ }^{1} \mathrm{H}$ Nuclear magnetic resonance (NMR) (Spinsolve 60, Magritek, Wellington, New Zealand).

\section{Results and Discussion}

The SEM analysis showed that the MN-C was in the form of agglomerates of micron-sized irregularly shaped particles (Figure 1a). The rough and bulgy surface of $\mathrm{MN}-\mathrm{C}$ particles offered a large surface area of $258 \mathrm{~m}^{2} / \mathrm{g}$ and pore volume of $0.379 \mathrm{~cm}^{3} / \mathrm{g}$. The partial decomposition of the organic part of the precursor during annealing led to a partial reduction of iron oxide nanoparticles to $\mathrm{Fe}^{0}$. A quantitative phase analysis based on the Rietveld refinement of the XRD pattern (Figure S3) showed that the MN-C consists of body centered cubic Fe nanoparticles $(26.0 \mathrm{wt} . \%$, with a crystallite size of $54 \mathrm{~nm}$ ), spinel iron oxide nanoparticles (10.0 wt.\%, $14 \mathrm{~nm}$ ) and nanocrystalline graphite ( $64.0 \mathrm{wt.} \%, 5 \mathrm{~nm}$ ). The total Fe content determined by XRF [19] was found to be $22.0 \mathrm{wt} . \%$. The STEM analysis of the $\mathrm{MN}-\mathrm{C}$ also revealed an irregular shape of the $\mathrm{MN}-\mathrm{C}$ and a homogeneous distribution of the magnetic nanoparticles within the carbon matrix (Figure S4). Raman spectroscopy showed that the carbon matrix consists of nanocrystalline graphite, amorphous carbon phases and polyenes (Figure S5) [20-25]. The MN-C showed ferromagnetic behavior with a high saturation magnetization $M_{\mathrm{S}}(\mathrm{Fe})=148 \mathrm{Am}^{2} / \mathrm{kg}_{\mathrm{Fe}}$ (expressed per amount of Fe) (Figure 1b). The good magnetic properties, namely, high magnetization and relatively high magnetic susceptibility (Figure 1b), were reflected in the high SAR values (measured at $273 \mathrm{kHz}$ ) (Figure 1c). (For details, see Supplementary Materials Section S2, Figures S1, S2 and Table S2). The SAR increased with the AC-field amplitude non-linearly, showing an abrupt increase above approximately $65 \mathrm{mT}$ and indicating a saturation above approximately $85 \mathrm{mT}$ (Figure 1c). The highest SAR value of approximately $800 \mathrm{~W} / \mathrm{g}_{\mathrm{Fe}}$ was measured for the sample at an amplitude of $97 \mathrm{mT}$ (Figure 1c).

(a)

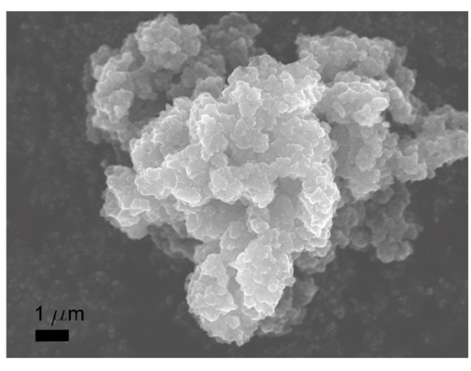

(b)

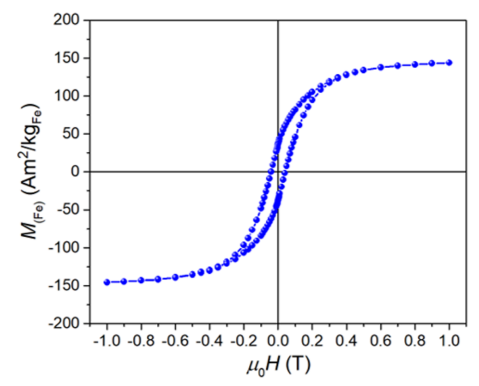

(c)

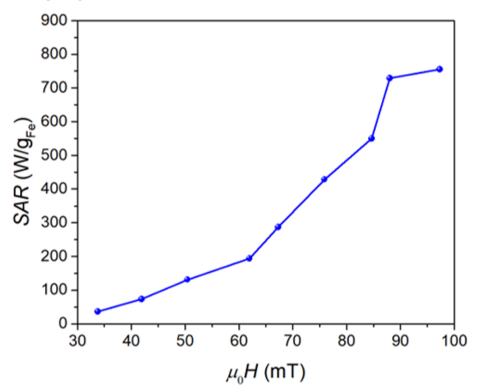

Figure 1. (a) SEM image of the room-temperature magnetization curve. (b) Specific absorption rate (SAR) (measured at $273 \mathrm{kHz}$ ) as a function of the AC-field amplitude (c) of the magnetic nanoparticle-containing support (MN-C).

The MN-C was used to demonstrate the magnetically mediated synthesis of the nanocatalyst AC-Ru (Figure 2). The synthesis was conducted in a closed quartz pressure vessel, with the MN-C particles dispersed in a solution of $\mathrm{Ru}\left(\mathrm{C}_{5} \mathrm{H}_{7} \mathrm{O}_{2}\right)_{3}$ in 2-propanol (see Supplementary Materials Section S2). 
The vessel was inserted into an inductor coil and exposed to the AC-magnetic field with an amplitude of $\mu_{0} H=86 \mathrm{mT}$ at the frequency of $273 \mathrm{kHz}$. No stirring was applied, and the temperature of the liquid was monitored by measuring the wall temperature of the vessel with the IR camera. Within 19 min (Table S1), practically the entire $\mathrm{Ru}^{3+}$ was reduced and deposited on the surface of the support in an exceptionally homogeneous manner (Figure $2 a, b$ ). To check if the only source of heat was magnetic MN-C particles, the blank test was performed. The same amount of isopropanol was positioned at the same position within the coils as the MN-C sample. Applying the same AC-field amplitude (frequency of $273 \mathrm{kHz}$ ) for $30 \mathrm{~min}$ resulted in a minor, $3{ }^{\circ} \mathrm{C}$ increase of the temperature, indicating that the coils or any other part of the equipment do not contribute to the heating of the MN-C. Recently, it was shown that the metastable tetragonal Ru exhibits ferromagnetic behavior [26]. Comparing the SAR values (unpublished results) of similar materials containing Ru nanoparticles with bare ones did not show any measurable difference, indicating that $\mathrm{Ru}$ nanoparticles do not contribute to heating.

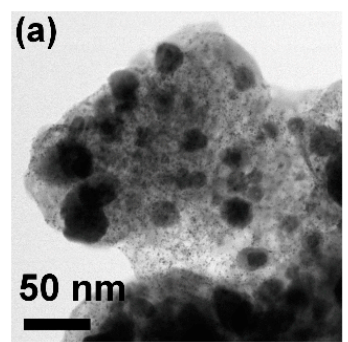

(e)

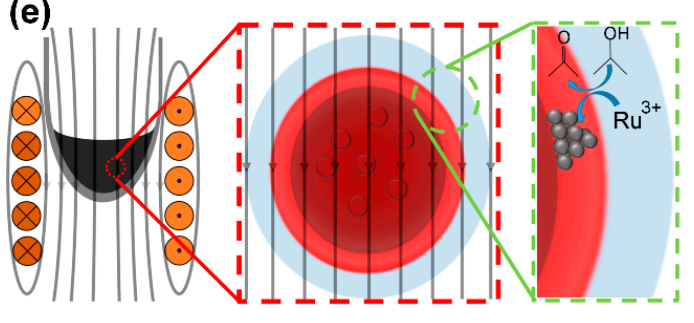

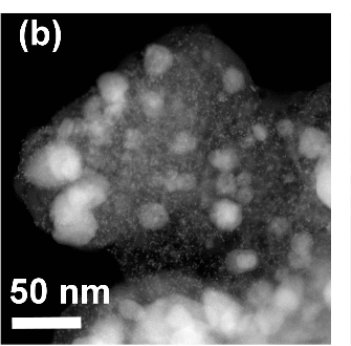
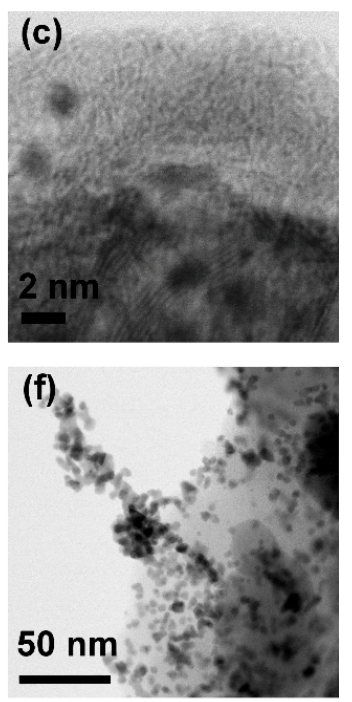
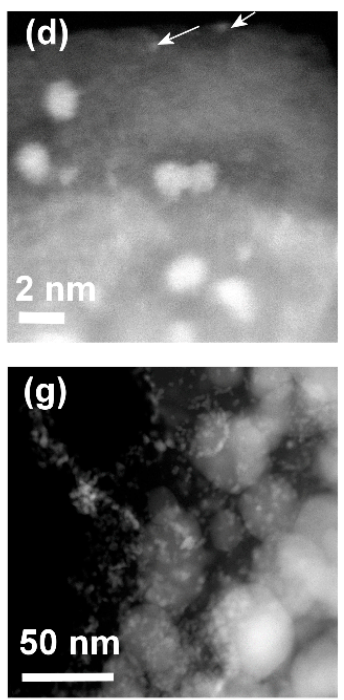

Figure 2. (a,c) Bright Field (BF) and (b,d) High Angle Annular Dark Field (HAADF) scanning transmission electron microscope (STEM) images of the nanocatalyst AC-Ru. (e) Schematic illustration of the magnetically mediated synthesis of the nanocatalyst AC-Ru. (f) BF and (g) HAADF STEM images of the nanocatalyst $\mathrm{CH}-\mathrm{Ru}$. Arrows in (d) show Ru cluster.

The size distribution of the Ru nanoparticles was narrow, with an average diameter of $\mathrm{d}=1.6 \pm 0.4 \mathrm{~nm}$ (Figure 2c,d). Free Ru nanoparticles (not attached to the support) were not observed. In addition to nanoparticles, clusters of $\mathrm{Ru}$ atoms were also formed (marked in Figure $2 \mathrm{~d}$ with arrows). The measured amount of elemental $\mathrm{Ru}(5.3 \mathrm{wt} . \%)$ in $\mathrm{AC}-\mathrm{Ru}$ is identical to the amount calculated from the mass balance, which indicates a complete reduction of $\mathrm{Ru}^{3+}$. In the magnetically mediated synthesis of the AC-Ru, the temperature of the liquid never exceeded $100{ }^{\circ} \mathrm{C}$ (Table S1). Our unpublished study showed that the reduction of $\mathrm{Ru}\left(\mathrm{C}_{5} \mathrm{H}_{7} \mathrm{O}_{2}\right)_{3}$ in 2-propanol proceeds only above $130{ }^{\circ} \mathrm{C}$. The above suggests that the surface of the $\mathrm{MN}-\mathrm{C}$ had rapidly been heated to a temperature above $130{ }^{\circ} \mathrm{C}$ and that the relatively thin film of liquid surrounding the heated particles remained at a high temperature, so $\mathrm{Ru}\left(\mathrm{C}_{5} \mathrm{H}_{7} \mathrm{O}_{2}\right)_{3}$ was completely reduced to $\mathrm{Ru}^{0}$ and nucleated exclusively on the surface as depicted in Figure 2e. For comparison, the nanocatalyst $\mathrm{CH}-\mathrm{Ru}$ was synthesized using conventional heating (see Supplementary Materials Section S1). The pressure vessel and its content were heated to $150{ }^{\circ} \mathrm{C}$ using an oil bath (see Supplementary Materials Section S1). The solution became colorless after $30 \mathrm{~min}$, indicating a slower reduction kinetics as in the case of the magnetically mediated synthesis of AC-Ru. The slower kinetics further suggests that the surface must have been heated above $150{ }^{\circ} \mathrm{C}$ during the synthesis of $\mathrm{AC}-\mathrm{Ru}$. Ru nanoparticles with a diameter of $\sim 3 \mathrm{~nm}$ were homogeneously distributed over the surface of $\mathrm{CH}-\mathrm{Ru}$ (Figure $2 \mathrm{f}, \mathrm{g}$ ). In addition to the individual nanoparticles, agglomerates of the Ru 
nanoparticles were also formed, which indicate homogeneous nucleation of the Ru nanoparticles in the bulk of the heated 2-propanol (Figure 2f,g and Figure S6). The weight fraction of the deposited Ru in the $\mathrm{CH}-\mathrm{Ru}$ was $\sim 3 \mathrm{wt} . \%$, lower than expected from the mass balance, suggesting that a significant fraction of the $\mathrm{Ru}$ nanoparticles was removed during washing of the $\mathrm{CH}-\mathrm{Ru}$ after synthesis.

The catalytic test in a pressurized stirred slurry reactor showed a very high activity of AC-Ru nanocatalyst for furfural hydrotreatment in 2-propanol. Furfural that is produced from xylose is regarded as a versatile and renewable chemical with a wide range of industrial applications [27-30]. Conversion to the furfuryl alcohol compound used mainly in the polymer industry is a simple catalyzed hydrogenation reaction that consumes nearly $60 \%$ of furfural produced worldwide [27-30]. In the first run, the conversion of furfural over AC-Ru was 100\% in $3 \mathrm{~h}$, even at a very low reaction temperature of $90^{\circ} \mathrm{C}$, while selectivity for furfuryl alcohol was $80 \%$ (Figure 3). The side products were tetrahydrofurfuryl alcohol (18\%) and methyl furan ( $2 \%)$, indicating very high activity of the AC-Ru. Recyclability of the AC-Ru was tested in another four consecutive runs. Conversion remained at 100\%, but selectivity for furfuryl alcohol increased to $99.1 \%$ in the fifth run (Figure 3), demonstrating high activity of AC-Ru for aldehyde group hydrogenation, while it kept the five-membered heterocyclic ring intact. The high activity and selectivity of the AC-Ru strongly suggest that the metallic Ru nanoparticles were the catalytically active phase [27-30]. The increase of selectivity was most likely related to a slight decrease of activity in the most active sites responsible for over hydrogenation and dehydration. Catalytic activity of the $\mathrm{CH}-\mathrm{Ru}$ was, despite lower fraction of $\mathrm{Ru}$, remarkably good. In the first run, the conversion was $100 \%$ and selectivity for the furfuryl alcohol was $95 \%$ (Figure S7). Detailed spectrometric investigation ( $\mathrm{H}^{1} \mathrm{NMR}$ and QMS) of the products revealed that the remaining $5 \mathrm{~mol} \%$ of the products was formed via the competitive reaction between furfural and isopropanol in the bulk liquid; specifically, acetalization reaction took place yielding furfural diisopropyl acetal (CAS 187995-47-7). Its formation was detected in blank test runs in absence of the catalyst; therefore, its formation in the bulk liquid could only be further reduced with an increase of the catalyst loading or completely omitted using a suitable solvent without the hydroxyl functional group. The catalyst $\mathrm{CH}-\mathrm{Ru}$ remained active in the second run with similar selectivity and product distribution (Figure S7).

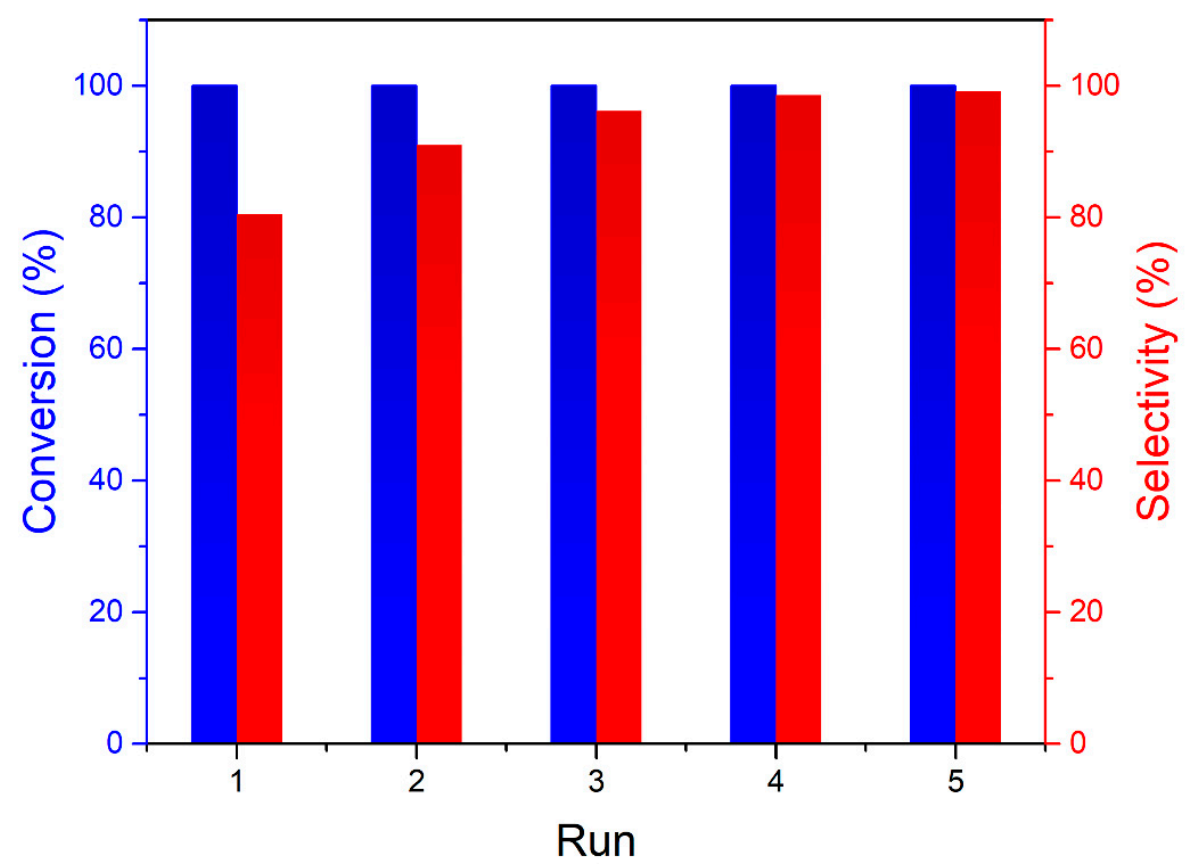

Figure 3. Conversion of furfural and selectivity for the furfuryl alcohol over the AC-Ru nanocatalyst in five consecutive runs. (More details in Supplementary Materials Section S1) Reaction conditions: AC-Ru (48 mg), furfural (0.1 g, $1.04 \mathrm{mmol}), 2$-propanol $(0.8 \mathrm{~mL}), \mathrm{H}_{2}, 1 \mathrm{MPa}, 90{ }^{\circ} \mathrm{C}, 3 \mathrm{~h}$. 


\section{Conclusions}

In conclusion, we have shown a magnetically mediated process for the rapid synthesis of a $\mathrm{Ru}$-based nanocatalyst. The locally delivered heat led to homogeneous deposition of Ru nanoparticles with a diameter of $1.6 \mathrm{~nm}$ exclusively on the surface of the support. Compared to conventional heating, the synthesis time for nanocatalyst preparation was significantly shortened; the magnetically mediated heating up period was practically immediate; the time to quantitatively deposit Ru was significantly decreased and the cooling down period was immediate, since the bulk of the liquid medium remained at a significantly lower temperature. The process, although far from being optimized, offers utilization of electricity for the rapid synthesis of this functional material. The synthesized nanocatalyst showed remarkable activity in the hydrogenation of furfural to furfuryl alcohol. After four recycling cycles, the conversion remained at $100 \%$, while selectivity was steadily increased from $80 \%$ in the first run to $>99 \%$ in the last.

Supplementary Materials: The following are available online at http://www.mdpi.com/2079-4991/10/6/1142/s1, Table S1: Vessel's surface temperature, pressure and solution color as the function of time into AC-field heating, Figure S1: Vector representation of the $\mu_{0} \mathrm{H}$ on a plane through the center of the inductor coil, Figure S2: Color map of the $\mu_{0} \mathrm{H}$ magnitude on the plane through the center of the coil, Figure S3: XRD powder pattern of the MN-C, Table S2: Estimated crystallite size and weight fraction of the corresponding crystalline phase, room-temperature magnetic properties and specific surface area of the MN-C, Figure S4: BF and HAADF STEM images of the MN-C, Figure S5: Raman spectrum of the MN-C, Figure S6: BF and HAADF STEM images of the CH-Ru, Figure S7: Recycling of the CH-Ru nanocatalyst.

Author Contributions: Conceptualization, S.G. and D.M.; methodology, S.G.; investigation, S.G., D.L., M.B., M.G., B.L., M.N. and D.M.; writing-original draft preparation, S.G.; writing-review and editing, S.G., M.G., B.L. and D.M.; supervision, S.G. and D.M.; funding acquisition, D.M., M.G. and B.L. All authors have read and agreed to the published version of the manuscript.

Funding: This research was funded by the Slovene Research Agency, grant numbers: P2-0089, P2-0152 and Z2-9200; FPS COST Action FP1306.

Acknowledgments: The authors acknowledge the CENN Nanocenter for the use of their Transmission Electron Microscope Jeol JEM-2100 and the Vibrating Sample Magnetometer LakeShore 7400 Series VSM. The authors would like to thank Andraž Kocjan from JSI for surface area measurements. The authors of this study are grateful to Peter Kump for kindly providing the QAES (Quantitative Analysis of Environmental Samples) software.

Conflicts of Interest: The authors declare no conflicts of interest.

\section{References}

1. She, Z.W.; Kibsgaard, J.; Dickens, C.F.; Chorkendorff, I.; Nørskov, J.K.; Jaramillo, T.F. Combining theory and experiment in electrocatalysis: Insights into materials design. Science 2017, 355, eaad4998.

2. Johannsen, M.; Gneveckow, U.; Thiesen, B.; Taymoorian, K.; Cho, C.H.; Waldöfner, N.; Scholz, R.; Jordan, A.; Loening, S.A.; Wust, P. Thermotherapy of prostate cancer using magnetic nanoparticles: Feasibility, imaging, and three-dimensional temperature distribution. Eur. Urol. 2007, 52, 1653-1662. [CrossRef]

3. Pollert, E.; Veverka, P.; Veverka, M.; Kaman, O.; Zaveta, K.; Vasseur, S.; Epherre, R.; Goglio, G.; Duguet, E. Search of new core materials for magnetic fluid hyperthermia: Preliminary chemical and physical issues. Prog. Solid State Chem. 2009, 37, 1-14. [CrossRef]

4. Ceylan, S.; Friese, C.; Lammel, C.; Mazac, K.; Kirschning, A. Inductive heating for organic synthesis by using functionalized magnetic nanoparticles inside microreactors. Angew. Chemie Int. Ed. 2008, 47, 8950-8953. [CrossRef] [PubMed]

5. Hartwig, J.; Ceylan, S.; Kupracz, L.; Coutable, L.; Kirschning, A. Heating under high-frequency inductive conditions: Application to the continuous synthesis of the neurolepticum Olanzapine (Zyprexa). Angew. Chemie Int. Ed. 2013, 52, 9813-9817. [CrossRef] [PubMed]

6. Chaudhuri, S.R.; Hartwig, J.; Kupracz, L.; Kodanek, T.; Wegner, J.; Kirschning, A. Oxidations of allylic and benzylic alcohols under inductively-heated flow conditions with gold-doped superparamagnetic nanostructured particles as catalyst and oxygen as oxidant. Adv. Synth. Catal. 2014, 356, 3530-3538. [CrossRef] 
7. Meffre, A.; Mehdaoui, B.; Connord, V.; Carrey, J.; Fazzini, P.F.; Lachaize, S.; Respaud, M.; Chaudret, B. Complex nano-objects displaying both magnetic and catalytic properties: A proof of concept for magnetically induced heterogeneous catalysis. Nano Lett. 2015, 15, 3241-3248. [CrossRef]

8. Bordet, A.; Lacroix, L.M.; Fazzini, P.F.; Carrey, J.; Soulantica, K.; Chaudret, B. Magnetically induced continuous $\mathrm{CO}_{2}$ hydrogenation using composite iron carbide nanoparticles of exceptionally high heating power. Angew. Chemie Int. Ed. 2016, 55, 15894-15898. [CrossRef]

9. Wang, W.; Tuci, G.; Duong-Viet, C.; Liu, Y.; Rossin, A.; Luconi, L.; Nhut, J.-M.; Nguyen-Dinh, L.; Pham-Huu, C.; Giambastiani, G. Induction heating: An enabling technology for the heat management in catalytic processes. ACS Catal. 2019, 9, 7921-7935. [CrossRef]

10. Niether, C.; Faure, S.; Bordet, A.; Deseure, J.; Chatenet, M.; Carrey, J.; Chaudret, B.; Rouet, A. Improved water electrolysis using magnetic heating of FeC-Ni core-shell nanoparticles. Nat. Energy 2018, 3, 476-483. [CrossRef]

11. Li, H.; Sadiq, M.M.; Suzuki, K.; Falcaro, P.; Hill, A.J.; Hill, M.R. Magnetic induction framework synthesis: A general route to the controlled growth of metal-organic frameworks. Chem. Mater. 2017, 29, 6186-6190. [CrossRef]

12. Melag, L.; Sadiq, M.M.; Smith, S.J.D.; Konstas, K.; Suzuki, K.; Hill, M.R. Efficient delivery of oxygen: Via magnetic framework composites. J. Mater. Chem. A 2019, 7, 3790-3796. [CrossRef]

13. Besson, M.; Gallezot, P.; Pinel, C. Conversion of biomass into chemicals over metal catalysts. Chem. Rev. 2014, 114, 1827-1870. [CrossRef] [PubMed]

14. Yang, Y.; Chiang, K.; Burke, N. Porous Carbon-supported catalysts for energy and environmental applications: A short review. Catal. Today 2011, 178, 197-205. [CrossRef]

15. Raghavan, N.; Thangavel, S.; Venugopal, G. A short review on preparation of graphene from waste and bioprecursors. Appl. Mater. Today 2017, 7, 246-254. [CrossRef]

16. Gao, Z.; Zhang, Y.; Song, N.; Li, X. Biomass-derived renewable carbon materials for electrochemical energy storage. Mater. Res. Lett. 2017, 5, 69-88. [CrossRef]

17. Gyergyek, S.; Kocjan, A.; Bjelić, A.; Grilc, M.; Likozar, B.; Makovec, D. Magnetically separable Ru-based nano-catalyst for the hydrogenation/hydro-deoxygenation of lignin-derived platform chemicals. Mater. Res. Lett. 2018, 6, 426-431. [CrossRef]

18. Bjelić, A.; Grilc, M.; Gyergyek, S.; Kocjan, A.; Makovec, D.; Likozar, B. Catalytic hydrogenation, hydrodeoxygenation, and hydrocracking processes of a lignin monomer model compound eugenol over magnetic $\mathrm{Ru} / \mathrm{C}-\mathrm{Fe}_{2} \mathrm{O}_{3}$ and mechanistic reaction microkinetics. Catalysts 2018, 8, 425. [CrossRef]

19. Nečemer, M.; Kump, P.; Ščančar, J.; Jaćimović, R.; Simčič, J.; Pelicon, P.; Budnar, M.; Jeran, Z.; Pongrac, P.; Regvar, M.; et al. Application of X-ray fluorescence analytical techniques in phytoremediation and plant biology studies. Spectrochim. Acta Part B 2008, 63, 1240-1247. [CrossRef]

20. Ferrari, A.C.; Robertson, J. Raman spectroscopy of amorphous, nanostructured, diamond-like carbon, and nanodiamond. Philos. Trans. R. Soc. Lond. Ser. A. 2004, 362, 2477-2512. [CrossRef]

21. Cançado, L.G.; Pimenta, M.A.; Neves, B.R.A.; Dantas, M.S.S.; Jorio, A. Influence of the atomic structure on the raman spectra of graphite edges. Phys. Rev. Lett. 2004, 93, 5-8. [CrossRef]

22. Cuesta, A.; Dhamelincourt, P.; Laureyns, J.; Martínez-Alonso, A.; Tascón, J.M.D. Raman microprobe studies on carbon materials. Carbon 1994, 32, 1523-1532. [CrossRef]

23. Jawhari, T.; Roid, A.; Casado, J. Raman spectroscopic characterization of some commercially available carbon black materials. Carbon 1995, 33, 1561-1565. [CrossRef]

24. Dippel, B.; Jander, H.; Heintzenberg, J. NIR FT Raman spectroscopic study of flame soot. Phys. Chem. Chem. Phys. 1999, 1, 4707-4712. [CrossRef]

25. Mallet-Ladeira, P.; Puech, P.; Toulouse, C.; Cazayous, M.; Ratel-Ramond, N.; Weisbecker, P.; Vignoles, G.L.; Monthioux, M. A Raman study to obtain crystallite size of carbon materials: A better alternative to the Tuinstra-Koenig law. Carbon 2014, 80, 629-639. [CrossRef]

26. Quarterman, P.; Sun, C.; Garcia-Barriocanal, J.; DC, M.; Lv, Y.; Manipatruni, S.; Nikonov, D.E.; Young, I.A.; Voyles, P.M.; Wang, J.P. Demonstration of $\mathrm{Ru}$ as the 4th ferromagnetic element at room temperature. Nat. Commun. 2018, 9, 2058. [CrossRef]

27. Wang, Y.; Zhao, D.; Rodríguez-Padrón, D.; Len, C. Recent advances in catalytic hydrogenation of furfural. Catalysts 2019, 9, 796. [CrossRef] 
28. O'Driscoll, A.; Leahy, J.J.; Curtin, T. The influence of metal selection on catalyst activity for the liquid phase hydrogenation of furfural to furfuryl alcohol. Catal. Today 2017, 279, 194-201. [CrossRef]

29. Lucarelli, C.; Bonincontro, D.; Zhang, Y.; Grazia, L.; Renom-Carrasco, M.; Thieuleux, C.; Quadrelli, E.A.; Dimitratos, N.; Cavani, F.; Albonetti, S. Tandem hydrogenation/hydrogenolysis of furfural to 2-methylfuran over a Fe/Mg/O catalyst: Structure-activity relationship. Catalysts 2019, 9, 895-910. [CrossRef]

30. Gupta, N.; Dimitratos, N.; Su, D.; Villa, A. Valorisation of biomass derived furfural and levulinic acid by highly efficient Pd@ND catalyst. Energy Technol. 2019, 7, 269-276. [CrossRef]

(C) 2020 by the authors. Licensee MDPI, Basel, Switzerland. This article is an open access article distributed under the terms and conditions of the Creative Commons Attribution (CC BY) license (http://creativecommons.org/licenses/by/4.0/). 